\title{
eJRIEPS
}

Ejournal de la recherche sur l'intervention en éducation physique et sport

$50 \mid 2022$

Varia

\section{Stratégie d'évaluation permettant des choix d'activités physiques aux étudiants : impact sur leur motivation pour un cours d'éducation physique au postsecondaire}

Assessment strategy allowing students to choose physical activity : impact on their motivation for a post-secondary physical education course

\section{Annie Bradette et Isabelle Cabot}

\section{OpenEdition}

\section{Journals}

Édition électronique

URL : https://journals.openedition.org/ejrieps/7697

DOI : 10.4000/ejrieps.7697

ISSN : 2105-0821

Éditeur

ELLIADD

Édition imprimée

Pagination : $42-79$

Référence électronique

Annie Bradette et Isabelle Cabot, « Stratégie d'évaluation permettant des choix d'activités physiques aux étudiants : impact sur leur motivation pour un cours d'éducation physique au postsecondaire», eJRIEPS [En ligne], 50 | 2022, mis en ligne le 10 janvier 2022, consulté le 12 janvier 2022. URL : http:// journals.openedition.org/ejrieps/7697 ; DOI : https://doi.org/10.4000/ejrieps.7697

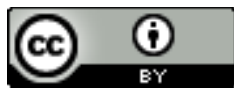

La revue eJRIEPS est mise à disposition selon les termes de la Creative Commons Attribution 4.0 International License. 


\section{Stratégie d'évaluation permettant des choix d'activités physiques aux étudiants :} impact sur leur motivation pour un cours d'éducation physique au postsecondaire

Annie Bradette* \& Isabelle Cabot**

* Cégep Édouard-Montpetit, Canada.

${ }^{*}$ Cégep Saint-Jean-sur-Richelieu, Canada ; Université de Sherbrooke, Canada.

Résumé

L'adolescence est un passage crucial où l'on observe un désengagement à la pratique d'activités physiques (AP) et une démotivation envers les cours d'éducation physique et à la santé (EPS) au secondaire et au postsecondaire, entre autres, dû aux évaluations de type traditionnel. Néanmoins, le milieu scolaire est un lieu privilégié pour promouvoir un mode de vie sain et actif par les cours d'EPS. Dans le but d'augmenter la motivation à l'égard des cours d'EPS du postsecondaire, une stratégie d'évaluation terminale, permettant aux étudiants (âge moyen :19) de faire un choix de test cardiorespiratoire, a été testée $(n=34)$ à travers un devis quasi-expérimental "prétest/posttest " avec condition témoin $(n=45)$. Les principaux résultats ont révélé une augmentation significative de l'intérêt suscité par le cours, ainsi qu'une augmentation de la contrôlabilité perçue sur les modalités d'évaluation du cours. Une analyse de contenus d'entrevues $(n=11)$ a révélé que d'offrir un choix de test cardiorespiratoire permettant aux étudiants d'exprimer leurs préférences en termes d'AP avait eu une influence favorable sur leur pratique d'AP en général, en dehors de la classe. Ces résultats invitent les enseignants d'EPS à instaurer des pratiques évaluatives innovantes permettant une certaine autonomie aux adolescents et jeunes adultes.

Mots clés : éducation physique et à la santé (EPS), stratégie d'évaluation, contrôlabilité, intérêt, postsecondaire 
Assessment strategy allowing students to choose physical activity: impact on their motivation for a post-secondary physical education course

\section{Summary}

Adolescence is a critical juncture where we observe a disengagement from the practice of physical activities (PA) and a demotivation towards physical education (PE) courses in secondary and post-secondary students due, inter alia, to traditional type evaluations. Nevertheless, the school environment is a privileged place to promote a healthy and active lifestyle through PE lessons. To increase motivation towards postsecondary PE courses, a final evaluation strategy allowing students (mean age: 19) to choose a cardiorespiratory test ( $n=34$ ) was tested through a quasi-experimental design with pretest/posttest measures and a control group $(n=45)$. The main results revealed a significant increase in perceived controllability over the course evaluation modalities as well as an increase in interest in the course. Analysis of interview content $(n=11)$ found that offering a choice of cardiorespiratory testing that allowed students to express their PA preferences had a positive influence on their PA practice in general, outside the class. These results invite $P E$ teachers to introduce innovative assessment practices that allow adolescents and young adults a certain autonomy.

Key words: physical education, assessment strategy, controllability, interest, postsecondary

\section{Introduction}

Selon le comité scientifique de Kino-Québec (2012), on observe une importante diminution de la motivation pour les cours d'éducation physique et à la santé (EPS) durant l'adolescence. Par exemple, Martins \& al. (2015) rapportent que 90\% des filles de 15 ans pratiquent moins de 60 minutes d'activités physiques par jour. Ensuite, le passage au postsecondaire mène les étudiants vers des changements caractéristiques du mode de vie puisqu'ils font face à une autonomie émergente en s'appropriant une grande liberté décisionnelle ainsi qu'une flexibilité d'horaire découlant du contexte postsecondaire (Ministère de l'Enseignement supérieur, de la Recherche, de la Science et de la Technologie [MERST], 2013 ; Ministère de l'Éducation et de l'Enseignement supérieur [MEES], 2019). Durant ces années d'entrée dans l'âge adulte, on rapporte un déclin de la condition physique des étudiants (Lemoyne \& Girard, 2019) qui pourrait s'expliquer en partie par un état de démotivation vis-à-vis de l'AP (Chiasson, 2004). 


\section{eJRIEPS 50 janvier 2022}

Au Québec, les collèges (nommés "cégeps " dans le secteur public) représentent le premier palier d'études postsecondaires et offrent des programmes d'études techniques, conduisant directement au marché du travail, et pré-universitaires (MEES, 2021). Près de la moitié de la population québécoise âgée de 25 à 64 ans détient un diplôme d'études collégial (Institut de la statistique du Québec, 2020). Les années au collégial se déroulent au tout début de l'âge adulte (autour de 17-20 ans). Elles constituent une période importante de développement et de changements physiologiques et psychologiques représentant un moment charnière qui sera déterminant dans la vie des collégiens (MERST, 2013; MEES, 2019). À cet âge, ils deviennent autonomes dans la gestion de leur temps entre les études, le travail et les activités personnelles (Bates, 2015, Nolin et Institut national de santé du Québec, 2018). Selon Gaudreault \& al., (2016), cette période de leur vie les rend plus vulnérables puisque les étudiants travaillent à un emploi rémunéré, en moyenne entre 15 et 19 heures par semaine. Trouver du temps à consacrer à la pratique régulière d'activités physiques (AP) peut représenter un réel défi pour cette clientèle scolaire (MERST, 2013; MEES, 2019). En contrepartie, il est reconnu que cette période de vie est propice au développement des habitudes de vie pouvant s'ancrer chez les étudiants, et ce, jusque durant l'âge adulte (MEES, 2019; Lemoyne, 2012; Menschik \& al., 2008). En EPS, la motivation est un élément fondamental à prendre en considération pour encourager les étudiants à adopter et maintenir un mode de vie actif (Pasco \& Spreux, 2014). Ainsi, motiver les étudiants dans les cours postsecondaires d'EPS est primordial.

Au Québec, le programme d'études collégiales de la formation générale est constitué de trois cours d'EPS obligatoires dans le cursus de l'étudiant. À travers ce parcours, le premier cours vise à ce que l'étudiant « analyse sa pratique de l'AP au regard des habitudes de vie favorisant la santé » (MEES, 2016, p. 28). Dans le second cours, l'étudiant doit « améliorer son efficacité dans la pratique d'une AP » (ibid., p. 29). Finalement, dans le dernier cours, l'étudiant doit " démontrer sa capacité à se charger de sa pratique de I'AP dans une perspective de santé » (ibid., p. 30). Tous les étudiants sont tenus de compléter avec succès leurs trois cours d'EPS en vue de l'obtention du diplôme d'études collégial, celui-ci donnant accès, soit aux professions techniques du marché du travail, soit aux études universitaires (ibid.). Le but premier des cours d'EPS au postsecondaire est d'encourager l'autonomie chez les étudiants afin de développer des citoyens responsables, autonomes et conscients de leurs choix au regard de leurs habitudes de vie (ibid.; Lemoyne, 2012). Ils sont les derniers cours d'EPS obligatoires du système scolaire québécois. 


\section{eJRIEPS 50 janvier 2022}

\section{Problématique}

De récentes déclarations de l'OMS (2019a) énoncent que la majorité des adolescents du monde ne sont pas suffisamment actifs, ce qui peut mettre en danger leur santé actuelle et future. Au niveau mondial, plus de $80 \%$ des adolescents scolarisés ne bougent pas assez (ibid.). Parallèlement, une diminution de l'intérêt des adolescents envers les cours d'EPS a été constatée (Chen \& Hancock, 2006; Lemoyne \& Girard, 2019; Robitaille, 2019). De plus, deux périodes charnières dans la diminution de la pratique d'AP ont été observées. Cellesci coïncident avec les périodes de transitions scolaires : l'une du primaire au secondaire (Kirk, 2005) et l'autre du secondaire au postsecondaire (Green, 2004). Ce dernier auteur précise que si l'étudiant réussit à maintenir son niveau de pratique de l'AP pendant ces deux périodes cruciales, il y a de fortes chances qu'il perpétue ces bonnes habitudes de vie dans le futur. Pour expliquer cette démotivation face à la pratique d'AP par les étudiants, il est évoqué qu'il y a une perte de plaisir en lien avec le contexte et les conditions de réalisation de l'AP (Kino-Québec, 2012). Finalement, les nouvelles responsabilités des adolescents qui les invitent à avoir un comportement dit plus « adulte » ou mature laissent moins de temps aux jeux, aux loisirs et aux AP (ibid., Martins et al., 2015). Toujours est-il qu'à l'adolescence, les étudiants ont un certain besoin de liberté et d'autonomie. Cela se concrétise par le désir de faire des choses pour eux-mêmes et tel qu'ils le souhaitent, comme faire des AP selon leurs intérêts personnels ou à un moment qui leur semble opportun. Fredricks \& al., (2004) mentionnent que ce besoin d'autonomie peut être respecté dans les milieux scolaires. Par exemple, un certain contrôle pourrait être offert à l'étudiant lui permettant de faire des choix d'AP ou de modalités d'évaluation dans les cours d'EPS. Ces conditions pourraient susciter l'intérêt des étudiants à adhérer à l'AP grâce à un besoin d'autonomie comblé.

\subsection{Démotivation envers l'AP : d'importantes conséquences}

Le désintéressement des adolescents envers l'AP ainsi qu'envers les cours d'EPS engendre certaines conséquences. D’une part, un impact direct est constaté sur le niveau de la condition physique des jeunes (Chiasson \& al., 2006; Lemoyne \& Girard, 2019) de même que sur le développement des maladies chroniques (les maladies cardiovasculaires, l'hypertension, le diabète de type 2, divers cancers et l'ostéoporose) (OMS, 2019b). Cette inactivité physique entraîne des retombées socio-économiques importantes (Avis du comité scientifique de Kino-Québec, 2020; OMS, 2019b). Entre autres, les comportements sédentaires imputent certains systèmes de santé publique puisque le coût mondial lié à l'inactivité physique correspond à 54 milliards de dollars par an (OMS, 2019b). De même, 


\section{eJRIEPS 50 janvier 2022}

un coût mondial indirect de l'inactivité physique correspondant à 14 milliards de dollars est observable en lien avec la perte de productivité, par exemple, par une diminution de l'efficacité et une augmentation du taux d'absentéisme (ibid.). D'autre part, des conséquences environnementales sont aussi liées à l'inactivité physique. En favorisant le transport actif à la marche ou à vélo, cela permettrait de diminuer l'utilisation de l'automobile. Ce geste réduirait les embouteillages, la pollution et l'émission de gaz à effet de serre (Mueller \& al., 2015). En somme, ces conséquences assujetties à la sédentarité pourraient être amoindries grâce à l'adhésion à la pratique d'AP par la majorité de la population de notre société (Avis du comité scientifique de Kino-Québec, 2020; OMS, 2019b).

\subsection{Des causes de l'inactivité physique}

Au postsecondaire, parmi les obstacles à la pratique d'AP, Chiasson \& al. (2006) évoquent le déclin de la condition physique des étudiants comme un facteur de démotivation envers la pratique de l'AP. À cet effet, il est intéressant de se questionner sur ce lien. Est-ce le déclin de leur condition physique qui démotive les étudiants à être actifs ou est-ce l'état de démotivation envers l'activité physique qui amène cette détérioration de la condition physique? Néanmoins, les causes de cette démotivation peuvent être multiples.

Brand (2015), énonce que différents types de morphologies peuvent influencer la motivation de l'étudiant à la pratique de l'AP. Par exemple, un étudiant en surpoids peut rencontrer certains obstacles personnels à la réalisation d'une tâche pédagogique en EPS. De plus, le comité scientifique de Kino-Québec (2014) met en lumière l'écart important entre les filles et les garçons dans la pratique d'AP et sportive et rappelle la problématique liée au désengagement des adolescentes vis-à-vis l'AP. Dans ce rapport, on propose de considérer les besoins et champs d'intérêt dans la planification des AP.

De plus, l'évaluation en EPS représente un défi de taille pour les enseignants et pourrait représenter une autre cause de l'inactivité physique de certains étudiants. Dans les années 1990, Carroll (1994) estimait que les tests de la condition physique représentaient environ $90 \%$ des évaluations utilisées en EPS et critiquait leur utilisation à la lumière d'éléments comme la génétique et la motivation. En effet, l'évaluation de la condition physique par des tests standardisés utilisant des critères normatifs de performance aurait avantage à être modifiée pour une approche axée sur le processus d'apprentissage de l'étudiant envers sa condition physique (Pollock \& al., 1998; The Cooper Institute, 2017). Cela permettrait d'éviter de comparer les étudiants entre eux, ce qui favoriserait un climat positif face à l'AP ainsi que l'engagement des étudiants en EPS (The Cooper Institute, 2017). Malgré cela, ce 


\section{eJRIEPS 50 janvier 2022}

type d'évaluation traditionnel demeure aujourd'hui très répandu au Québec (Bradette \& Cabot, 2019) et ailleurs dans le monde (Lopez-Pastor \& al., 2013). Une approche plus convenable et pédagogique pourrait être privilégiée afin d'établir de meilleures pratiques dans les milieux scolaires collégiaux.

En conséquence, il est pertinent, en tant que pédagogue, de considérer ces réalités afin d'adapter l'enseignement pour mener les étudiants à persévérer dans leur pratique d'AP. Ainsi, en veillant à contribuer à combler le besoin d'autonomie des étudiants, il serait possible d'observer un engagement envers le cours (Fredricks \& al., 2004). En effet, différents modèles de la motivation soutiennent l'idée que la contrôlabilité, ou la satisfaction du besoin d'autonomie, influence positivement la motivation à s'engager et à persévérer (Ames, 1992; Ryan \& Deci, 2000; Viau, 2009).

\subsection{Promouvoir l'AP par les cours d'EPS : rôle de l'éducation physique}

L'OMS (2019b) présente les cours d'EPS comme étant une ressource prioritaire pour promouvoir l'AP. II propose de renforcer l'offre de cours tout en essayant d'augmenter les expériences positives chez les étudiants, peu importe leur niveau scolaire. De son côté, le Gouvernement du Québec a contribué à la mise sur pied d'une Chaire de recherche (appelée Kino-Québec) s'intéressant aux aspects entourant l'adoption d'un mode de vie physiquement actif en contexte scolaire (Université de Sherbrooke, 2018). L'un de ses objectifs concerne le développement et le soutien d'interventions pédagogiques efficaces et prometteuses.

D'ailleurs, la littérature scientifique traitant des situations d'intervention stimulant la pratique d'AP énonce que le milieu scolaire est un terreau fertile à l'enseignement des saines habitudes de vie (Gadais, 2015; MEES, 2019; OMS, 2019b). Les cours d'EPS, en plus d'être obligatoires, permettent de sensibiliser tous les jeunes de divers milieux sociaux en proposant des opportunités de pratique de l'AP en donnant accès à des ressources humaines, matérielles, ainsi qu'à des lieux physiques propices à l'apprentissage d'AP (Trost \& Loprinzi, 2008). Néanmoins, Chen \& Hancock (2006) spécifient que plusieurs interventions pédagogiques veulent influencer le niveau d'AP à défaut de susciter un changement de comportement visant à amener le développement d'un mode de vie actif et durable. Pour sa part, Kirk (2013) énonce que le transfert des apprentissages réalisés en EPS vers la vie adulte reste très peu significatif. Actuellement, il faut se questionner sur les conditions d'apprentissage des étudiants dans les cours d'EPS en vue de proposer des activés pédagogiques visant un développement propice à l'adoption d'un mode de vie actif 


\section{eJRIEPS 50 janvier 2022}

(Robitaille, 2019). Afin d'encourager ce changement de comportement durable, susciter la perception de contrôle des étudiants, dans une tâche évaluative en situation authentique, pour les motiver et provoquer l'engagement pourrait représenter une piste de solution.

Bien que les cours d'EPS soient ciblés comme étant un lieu privilégié à l'apprentissage des saines habitudes de vie, les contraintes d'évaluation, les AP imposées sans égard aux besoins, aux aptitudes et aux préférences de l'étudiant, ainsi que le climat de classe valorisant davantage la performance que le plaisir, engendrent des écueils à l'engagement de certains adolescents et jeunes adultes en EPS (Baril \& al., 2014; Robitaille, 2019). D'ailleurs, une étude récente portant sur la perception des élèves du dernier cycle du secondaire envers leur cours d'EPS rapporte que $74 \%$ d'entre eux déclarent qu'ils aiment I'EPS (Robitaille, 2019). Toutefois, les garçons et les filles ne manifestent pas tout à fait le même intérêt pour leurs cours d'EPS puisque, selon cette étude, $87 \%$ des garçons et seulement $60 \%$ des filles apprécient l'EPS. D'autant plus que $40 \%$ des filles ont un intérêt mitigé envers cette discipline bien qu'elles consentent à l'importance et l'utilité de cette matière pour la santé globale (Robitaille, 2019). Cette autrice conclut en récapitulant les perceptions des élèves envers le cours d'EPS, entre autres que les AP proposées dans les cours sont redondantes et que l'enseignement et les évaluations sont trop de types traditionnels.

Ces perceptions vont de pair avec les dires de Bélanger \& Tremblay (2012) ainsi que d'Howe \& Ménard (1993) qui affirment que l'évaluation des apprentissages au postsecondaire est une problématique récurrente. De même, la littérature internationale dépeint un portrait des pratiques évaluatives en EPS et exprime plusieurs difficultés d'évaluation vécues dans cette discipline précise (Grandchamp \& al., 2018). Par exemple, de trop nombreux enseignants recourent à des tests physiques standardisés qui peuvent être discriminatoires pour certains étudiants et en démotiver d'autres (Bradette \& Cabot, 2019; Lopès-Pastor \& al., 2013; 647, QCCS, 2012; The Cooper Institute, 2017). L'évaluation est un enjeu majeur parce qu'elle nécessite de mettre en lumière les apprentissages des étudiants le plus équitablement et objectivement possible (Grandchamp \& al., 2018). Dernièrement, d'autres écrits se questionnent sur les stratégies d'évaluation efficaces en EPS au postsecondaire comme les travaux de Lalime (2016) ainsi que de Messier (2016). Ces écrits actuels suggèrent des évaluations prometteuses en encourageant l'évaluation des apprentissages en situation authentique plutôt que l'unique performance.

Malgré l'évolution des pratiques évaluatives proposées depuis les dernières années, il existe encore beaucoup d'enseignants utilisant des méthodes d'évaluation dites 


\section{eJRIEPS 50 janvier 2022}

traditionnelles en EPS. Par exemple, les tests de la condition physique sont encore très populaires dans l'enseignement, bien que cette pratique ait été largement critiquée dans la littérature et que les étudiants en aient rapporté une expérience négative, démotivante et très peu d'apprentissages significatifs (Jaakkola \& al., 2013; Lopèz-Pastor \& al., 2013; Mercier \& Silverman, 2014; Wiersma e\&Sherman, 2008). En effet, bien qu'il soit documenté que les tests de la condition physique peuvent offrir certains avantages comme permettre de connaitre sa forme physique et de cibler les étudiants ayant une faible condition physique (Keating \& al., 2020) ces tests comportent aussi certains désavantages pouvant être nuisibles en fonction de leur utilisation (Cale \& al., 2014; Keating \& al., 2020). Parmi ces critiques, Keating \& al. (2020) soulignent que les tests de la condition physique sont parfois mal enseignés et que peu d'apprentissages significatifs sont faits par les étudiants. Mercier \& Silverman (2014) stipulent que les résultats axés sur la performance et non confidentiels peuvent impacter négativement l'attitude de certains étudiants envers l'AP. L'utilisation imposée des mêmes tests peut devenir ennuyeuse et répétive (ibid.). Ainsi, ces auteurs suggèrent d'enseigner et d'intégrer les tests de la condition physique sous forme d'évaluation appropriée afin de favoriser les apprentissages en EPS et minimiser les réactions indésirables. Au Québec, un sondage, mené auprès de 107 enseignants d'EPS au postsecondaire, indique que $68 \%$ d'entre eux utilisent les tests de la condition physique comme outil d'évaluation sommatif et 36 \% comme outil formatif (Bradette @ Cabot, 2019). La proposition d'évaluer les étudiants grâce à une méthode dite contemporaine permettant à l'étudiant de faire un choix de test cardiorespiratoire plutôt qu'imposé apparaît comme une solution envisageable. En effet, Viau (2009) affirme qu'en responsabilisant les étudiants dans leurs apprentissages en leur permettant de faire un choix, balisé par l'enseignant, favoriserait sa perception de contrôlabilité et sa motivation.

En considérant tout ce qui précède, trois choses devraient être examinées en parallèle. Premièrement, du côté des cours d'EPS, la stagnation des méthodes d'évaluation, observable dans le cours d'EPS depuis les dernières décennies (Robitaille, 2019). Deuxièmement, la baisse de motivation des étudiants envers les cours d'EPS lors du passage au postsecondaire. Troisièmement, la baisse de pratique d'AP observée durant la transition vers le jeune âge adulte, qui est une réelle préoccupation à l'échelle internationale. Ainsi, il semble judicieux de chercher à stimuler la motivation des étudiants du postsecondaire envers les cours d'EPS, par le biais d'une stratégie d'évaluation mieux ajustée aux connaissances récentes du domaine de la motivation. D'autant plus que, selon 
eJRIEPS 50 janvier 2022

Martins \& al., (2015), susciter la motivation des étudiants face aux cours d'EPS est une piste prometteuse et perçue comme un levier à la pratique régulière d'AP.

\section{Cadre conceptuel}

La plupart des écrits rapportant l'évaluation de l'impact motivationnel d'une stratégie pédagogique en EPS visent un impact sur la motivation à pratiquer l'AP. Très peu de recherches visent un impact sur la motivation ressentie à l'égard des cours d'EPS.

\subsection{La motivation affectée par les pratiques évaluatives et le climat de classe}

Durant l'étude de Robitaille (2019), 208 élèves du dernier cycle du secondaire (âgés de 15 à 18 ans) ont exprimé leurs perceptions sur quelques aspects pouvant susciter l'engagement envers leur cours d'EPS, notamment le type d'AP et la qualité de l'activité d'apprentissage proposée, le soutien de l'enseignant lors de l'apprentissage, la valorisation de la performance, ainsi que les stratégies d'apprentissage et d'évaluation utilisées. Au terme de l'étude, on conclut que les pratiques pédagogiques des enseignants d'EPS ne semblaient pas avoir évolué malgré plusieurs recommandations d'experts visant à motiver les étudiants. Ce manque de progrès en EPS influence négativement la motivation des élèves du secondaire. Ce désintérêt envers les activités pédagogiques proposées au secondaire est possiblement amoindri au niveau collégial, car les collégiens peuvent choisir, parmi quelques choix, la discipline sportive ou le moyen d'action par laquelle le cours obligatoire sera enseigné. Certaines pratiques évaluatives demeurent toutefois non conformes à ce qui est recommandé dans la littérature (Bradette \& Cabot, 2019; LópezPastor \& al., 2013), d'où la pertinence de mener des études où l'application de nouvelles stratégies d'évaluation par des enseignants sera expérimentée et évaluée.

Quant à Girard (2016), elle aborde le climat motivationnel en EPS en fonction des besoins psychologiques afin de mieux comprendre le phénomène de désengagement des adolescents face à l'AP. Des élèves du secondaire rapportaient un désengagement et une diminution de plaisir dans leurs cours d'EPS. Des raisons personnelles étaient évoquées, en plus de raisons liées à l'environnement scolaire. Par son étude, Girard (2016) a vérifié l'impact de ce climat sur les buts d'accomplissement, les attitudes et les habitudes des élèves du secondaire, auprès de 909 élèves âgés entre 15 et 18 ans. Les résultats confirment que les buts d'accomplissement des élèves se modifient selon le climat motivationnel perçu, ce qui influence le sentiment de compétence. Girard (2016) conclut que l'enseignant d'EPS a la possibilité d'agir sur la motivation ainsi que l'engagement des 


\section{eJRIEPS 50 janvier 2022}

élèves en cours et même en dehors des heures de classe. En s'assurant que l'environnement d'apprentissage réponde aux besoins des élèves, l'enseignant d'EPS aurait un impact sur leur motivation à être physiquement actifs.

\subsection{Actualiser les pratiques évaluatives pour susciter la motivation}

De leur côté, Leriche \& Walczack (2016) ont effectué une recherche précisément sur les pratiques des enseignants d'EPS visant à motiver les étudiants. Au total, 74 enseignants d'EPS du postsecondaire ont participé. Les résultats ont illustré que la vaste majorité des enseignants d'EPS organisent leurs cours afin de susciter le plaisir chez l'étudiant pour contribuer à la motivation de ces derniers à la pratique de l'AP. Également, des enseignants désirent concevoir de nouvelles stratégies pédagogiques et évaluatives favorisant l'appréciation et le rayonnement des apprentissages réalisés par les cours d'EPS, par exemple, une évaluation en situation authentique (en contexte réel) ou permettant aux étudiants de faire des choix d'AP évaluées favorisant le développement de l'autonomie. Le souhait derrière ces nouvelles stratégies est d'influencer favorablement les étudiants au maintien d'un mode de vie sain et actif au-delà des cours d'EPS obligatoires, mais dans un but ultime de contribuer à développer des citoyens en santé.

Par ailleurs, ce désir des enseignants d'EPS de susciter le plaisir des étudiants à pratiquer l'AP coïncide avec les travaux menés par Roure et ses collaborateurs sur le plaisir instantané et l'intérêt en situation d'AP (Roure \& Pasco, 2020). Jusqu'à présent, une des principales contributions de leurs travaux est certainement l'évaluation d'applications, en contextes réels d'EPS (p. ex. en badminton : Roure \& Pasco, 2017), et de connaissances théoriques sur l'intérêt en situation. Ces auteurs ont démontré que l'intérêt en situation des élèves pouvait être suscité en concevant des environnements éducatifs privilégiant le plaisir instantané, l'intention d'exploration et la demande d'attention (Roure \& Pasco, 2018 ; Roure, Kermarrec \& Pasco, 2019 ; Roure, Lentillon-Kaestner \& al., 2019 ; Roure, Méard \& al., 2019). Par conséquent, l'enseignant pourrait cibler un de ces aspects dans la conception des tâches pédagogiques et évaluatives afin d'engager les élèves à la pratique d'AP. Les auteurs soulignent le rôle primordial des enseignants dans la création des tâches d'apprentissages et d'évaluation afin de susciter l'intérêt en situation et l'engagement à long terme dans la pratique d'AP (ibid.).

\subsection{Des stratégies pédagogiques suscitant la motivation en EPS}




\section{eJRIEPS 50 janvier 2022}

Au Québec, Lemoyne (2012) s'est questionné sur l'apport des cours d'EPS précisément au postsecondaire. II a vérifié si les cours d'EPS encourageaient l'adoption d'un mode de vie sain et actif. Les résultats de son étude démontrent que c'est le troisième et dernier cours du programme d'EPS au collégial qui influence le plus positivement la pratique de l'AP au postsecondaire. Dans le but de favoriser l'adhésion à un mode de vie actif, il conclue son rapport de recherche, entre autres, en recommandant aux enseignants d'EPS de proposer la fixation d'objectifs orientés sur le processus plutôt que centrés sur le résultat et de miser sur les perceptions de soi afin de développer des activités pédagogiques augmentant le sentiment d'accomplissement tout en diminuant les situations de comparaison avec des normes idéales à obtenir (Lemoyne, 2012). De même, Lemoyne \& Girard (2019) ont évalué les trajectoires de changement des dimensions de l'estime de soi physique relatifs à la pratique d'activités physiques ainsi qu'au niveau de la condition physique chez des étudiants du postsecondaire. Cette étude en contexte naturel a assuré un suivi longitudinal, étalé sur une période de 18 mois, par 4 à 6 prises de mesure auprès d'un échantillon de 256 participants. Les résultats permettent de croire qu'une stratégie pédagogique visant à améliorer la condition physique et suscitant une pratique régulière d'AP aurait un impact favorable, particulièrement chez les étudiants sédentaires. Par exemple, les participants moins performants au départ ont connu les plus fortes évolutions des trajectoires de pratique d'AP de type cardiovasculaire.

En outre, concevoir une stratégie d'évaluation en EPS, visant à encourager le plaisir de bouger tout en évitant les comparaisons sociales et en encourageant l'amélioration personnelle, plutôt que l'atteinte de standards de performance, pourrait sembler une avenue intéressante. Ces conclusions sont en cohérence avec les résultats de recherche présentés par Leriche \& Walczak (2014) qui ont démontré que la motivation basée sur la compréhension et le plaisir de bouger représente une piste pédagogique intéressante en EPS. Elles s'accordent aussi avec des travaux de Chen \& al. (2001; 2014), menés aux États-Unis, dont les résultats confèrent un pouvoir particulier au plaisir instantané dans l'intérêt à pratiquer de l'AP.

Parallèlement, Lemoyne \& Valois (2012) suggèrent de développer des activités d'apprentissage ciblant l'augmentation de la perception de contrôle des étudiants face à leur pratique de l'AP. Ces auteurs ajoutent qu'il semble indispensable de se préoccuper des perceptions des étudiants à l'égard de leurs capacités physiques pour susciter la motivation à pratiquer de l'AP. Ils précisent que les activités pédagogiques présentées aux étudiants devraient être signifiantes et représenter un défi accessible tout en valorisant la réussite. 


\section{eJRIEPS 50 janvier 2022}

Au Royaume-Uni, Standage et ses collègues (2012) ont mené une étude basée sur la Théorie de l'autodétermination (Ryan et Deci, 2000) auprès de 494 élèves du secondaire. L'un des objectifs étaient de tester l'impact de l'autonomie vécue en cours d'EPS (exemple d'item : Dans ce cours d'EPS, j'ai la possibilité de faire des choix [traduction libre]) sur la motivation à faire de l'AP et sur l'AP mesurée objectivement à l'aide d'un podomètre. Les résultats ont indiqué une influence positive du soutien à l'autonomie en EPS sur la motivation à pratiquer de l'AP en dehors des cours, ainsi qu'un effet indirect (bien que modeste) sur la mesure objective d'AP. De leur côté, Haerens \& al., (2015), ont examiné la motivation perçue lors d'un enseignement en éducation physique favorisant le respect des besoins d'autonomie parallèlement au non-respect, et ce, sur 499 étudiants du secondaire. Au terme de cette étude, il est constaté que l'encouragement à l'autonomie lors des cours d'EPS suscite la motivation. Ces résultats ont donné un nouvel éclairage aux bénéfices que les étudiants peuvent retirer de l'influence pédagogique d'un enseignant supportant l'autonomie des étudiants en EPS.

\subsection{Le concept de la motivation}

Il existe plusieurs modèles théoriques abordant la motivation. Dans le cadre de la présente étude en contexte scolaire, une partie du modèle de la dynamique motivationnelle de Viau (2009) a été choisi, car il est spécifiquement adapté à la réalité pédagogique vécue en classe, et à son influence sur la motivation des étudiants. Les principaux déterminants de la motivation du modèle de Viau (2009) sont visés : la contrôlabilité, l'intérêt, l'utilité et le sentiment de compétence. Il est à noter que l'intérêt et l'utilité forment ensemble ce que Viau (2009) et d'autres chercheurs (p. ex. Wigfield \& Eccles, 2000) désignent comme la valeur attribuée à l'activité. Toutefois, Cabot (2017) a démontré que les variables de l'intérêt et de l'utilité sont deux construits différents et ne varient pas nécessairement ensemble. C'est pour cette raison que, dans la présente étude, ces deux variables sont présentées et mesurées distinctement.

\subsubsection{La contrôlabilité}

Selon Viau (2009), l'autonomie renvoie à la possibilité que l'étudiant a de faire des choix balisés par l'enseignant. Dans ce contexte, l'auteur utilise plutôt le terme " contrôlabilité " pour référer à la perception que l'étudiant a du contrôle qu'il peut exercer sur le déroulement d'une activité. II suggère que si les choix présentés aux étudiants sont en lien avec leurs intérêts et leurs buts, la contrôlabilité qu'ils peuvent ainsi exercer sur la tâche qu'ils doivent 


\section{eJRIEPS 50 janvier 2022}

faire les mènera à y attribuer plus d'utilité et à s'y sentir globalement plus motivé (Viau, 2009). Dans la perspective de la Théorie de l'autodétermination (Ryan \& Deci, 2000), fréquemment utilisée en recherche en EPS, on traduira que la contrôlabilité que l'enseignant offre aux étudiants permet de combler leur besoin fondamental d'autonomie. Donc, on peut croire qu'en augmentant la perception de contrôlabilité dans le cours d'EPS, par exemple en offrant aux étudiants la possibilité de choisir parmi des tâches pédagogiques à exécuter, cela devrait les mener à être plus motivés à l'égard du cours.

En effet, la possibilité de faire lui-même des choix, parmi des options disponibles ainsi que sur les actions elles-mêmes (Reeve \& al., 2003), permet à l'étudiant de mieux ajuster son expérience d'apprentissage à ses propres objectifs et comble son besoin d'autonomie, le motivant à s'engager davantage (Evans \& Boucher, 2015; Katz \& Assor, 2007). Du côté de la Théorie des buts d'accomplissement (Ames, 1992), on suggère aussi qu'un contexte d'apprentissage offrant des choix dans les tâches scolaires mène les étudiants à poursuivre des buts d'apprentissage, axés sur le développement de leurs compétences, plutôt que des buts de performance, axés sur la comparaison sociale (Patall \& Yang Hooper, 2019).

\subsubsection{L'intérêt}

L'intérêt est une variable motivationnelle très puissante, composée d'une part importante d'émotions en plus de sa composante cognitive (Hidi \& Renninger, 2006). Deux types d'intérêt sont distingués dans la littérature : l'intérêt en situation dépend du contexte dans lequel est effectuée la tâche, alors que l'intérêt individuel est un trait stable malgré les contextes dans lesquels la tâche est effectuée. Durant le développement d'un nouvel intérêt, il sera d'abord dépendant de la situation et davantage composé d'émotions que de cognitions. Puis, s'il est maintenu, il se cristallisera pour devenir un intérêt individuel et sa composante cognitive sera plus présente (Hidi \& Renninger, 2006). L'influence positive de l'intérêt sur l'engagement, l'apprentissage et l'atteinte d'objectifs durables est reconnue dans la littérature (Renninger \& Hidi, 2016; 2019; Cabot, 2012). Selon Alexander \& Jetton (1996), l'intérêt joue un rôle crucial dans le processus d'apprentissage et oriente en partie ce que l'étudiant choisit d'apprendre. En effet, l'intérêt de l'étudiant le motive et le conduit à un apprentissage significatif (Pasco \& Spreux, 2014; Roure, Kermarrec \& Pasco, 2019). Toutefois, l'intérêt individuel est déterminé par des connaissances, croyances ainsi que des valeurs individuelles, ce qui augmente le niveau de difficulté à concevoir un enseignement ou des tâches pédagogiques ciblant l'intérêt individuel de chacun. II est plutôt suggéré d'axer les interventions pédagogiques en EPS en fonction de susciter un intérêt en situation 


\section{eJRIEPS 50 janvier 2022}

(Roure, Lentillon-Kaestner \& al., 2019; Roure \& Pasco, 2017). Toutefois, Chen \& Wang (2017) appellent une meilleure compréhension des mécanismes pouvant faire basculer l'intérêt en situation vers un intérêt individuel pouvant être lié à l'intégration pérenne de l'AP dans un mode de vie sain.

Certains éléments influencent l'intérêt en situation des étudiants en EPS. D'une part, les facteurs individuels comme le genre et les habiletés motrices, et d'autre part, les facteurs contextuels comme la conception de la tâche d'apprentissage proposée par l'enseignant (Chen \& Darst, 2001; Pasco \& Spreux, 2014 ; Roure \& Pasco, 2017). L'intérêt en situation des étudiants peut donc être favorisé en manipulant les caractéristiques de la situation d'apprentissage (Lentillon-Kaestner \& Roure, 2019; Pasco \& al., 2015; Roure, LentillonKaestner \& al., 2019; Roure \& Pasco, 2020). Ainsi, une stratégie d'évaluation qui respecte les préférences des étudiants mènerait possiblement à susciter l'intérêt envers la tâche et même envers le cours d'EPS.

\subsubsection{L'utilité}

L'utilité attribuée à une tâche découle de l'évaluation de la compatibilité entre l'activité pédagogique (ou le cours) à réaliser et les objectifs visés par la personne (Wigfield \& Eccles, 2000). Ainsi, lorsque la tâche est considérée utile par l'étudiant en vue de l'atteinte de ses propres objectifs, la motivation et l'engagement de l'étudiant augmentent (Dubeau \& al., 2015). En somme, l'utilité est une variable motivationnelle de type cognitif qui a une grande influence sur l'engagement (Hulleman \& al., 2017). Jusqu'à présent, aucun résultat de recherche traitant spécifiquement de l'utilité attribuée aux cours d'EPS par les étudiants du postsecondaire n'a été trouvé dans la littérature. L'évaluation des compétences en situation authentique ainsi que la possibilité de faire des choix en fonction de leurs intérêts par les étudiants lors des situations d'évaluation pourraient être une solution envisageable afin d'encourager l'autonomie et de susciter l'utilité attribuée à la tâche ou au cours.

\subsubsection{La perception de compétence à faire de l'activité physique}

Pour Viau (2009), la perception que l'étudiant a de sa compétence à accomplir la tâche implique qu'avant d'entreprendre une activité qui comporte un niveau élevé d'incertitude quant à sa réussite, il évalue ses capacités à l'accomplir de manière adéquate. Cela suppose qu'un étudiant ayant une perception positive de sa compétence à réaliser une tâche pédagogique se verra accomplir cette dernière avec confiance (Viau \& al., 2004). De surcroît, plus l'étudiant a une perception positive de la tâche, plus il la réussira et par 


\section{eJRIEPS 50 janvier 2022}

conséquent plus son sentiment de compétence augmentera (Viau \& Bouchard, 2000; Wigfield \& Eccles, 2000). En contrepartie, si l'étudiant a échoué ou a eu une performance décevante, mais qu'il y a investi beaucoup d'effort, sa perception de compétence risque fort d'être ébranlée (Taylor \& al., 2010). Dans la littérature, plusieurs chercheurs arrivent au même consensus, soit que la perception de compétence joue un rôle très important dans l'état motivationnel et l'engagement (Ryan \& Deci, 2000; Taylor \& al., 2010; Viau, 2009; Wigfield \& Eccles, 2000), dans le cas présent, l'engagement dans la pratique d'AP (Lemoyne \& al., 2015).

\subsection{Objectif de la présente étude}

Durant la planification de cette étude, une intervention pédagogique a été élaborée de manière à tenir compte des éléments de la problématique et du cadre conceptuel présentés précédemment. II s'agit d'une stratégie d'évaluation qui devrait influencer, de manière globale, la dynamique motivationnelle des étudiants du postsecondaire à l'égard de leur cours d'EPS. Cette stratégie a été appliquée en contexte réel, dans le troisième cours d'EPS au collégial, qui représente la dernière occasion d'influence pédagogique du cheminement scolaire québécois en EPS. Cette stratégie implique de stimuler la contrôlabilité des étudiants par l'exercice d'un choix de test par lequel ils seront évalués, en fonction de leurs préférences d'AP (groupe expérimental). L'objectif principal de l'étude est de comparer leur motivation à l'égard du cours d'EPS à celles d'étudiants évalués de manière traditionnelle (groupe témoin), c'est-à-dire sans pouvoir exercer de contrôle sur la manière d'être évalués. Cette motivation (à l'égard du cours) a été évaluée par le biais de trois de ses déterminants : l'intérêt, l'utilité et la contrôlabilité, ressentis dans le contexte du cours d'EPS, ont été comparés ente les groupes en tenant compte des attentes d'intérêt, d'utilité et de contrôlabilité, mesurées au tout début du semestre. En complément, l'évolution du sentiment de compétence à pratiquer de l'AP, entre le début et la fin du semestre, a été comparée entre les groupes.

\section{Méthode}

Globalement, la présente étude quasi-expérimentale inclut des mesures prétest et posttest, avec condition témoin, ainsi qu'un devis d'analyse mixte. De plus, des précautions ont été prises pour contrôler certaines variables confondantes, notamment l'utilisation d'une application mobile, tant par les participants du groupe expérimental (GE) que par ceux du groupe témoin (GT), limitant ainsi l'impact de biais potentiels. 


\subsection{Participants}

Des étudiants provenant de deux établissements collégiaux québécois ont participé à l'étude à l'automne 2019. Dans chaque établissement, deux groupes-classes liés à un même enseignant étaient impliqués. Pour chacun de ces deux enseignants, un groupeclasse a vécu la condition expérimentale (décrite à la section 4.2). L'autre groupe-classe tenait le rôle de GT, c'est-à-dire que les étudiants de ce groupe suivaient le même cours, dans les mêmes conditions que ceux du GE du même enseignant, à l'exception de la condition expérimentale. II s'agit donc d'un échantillon de convenance. Plus précisément, 105 ( $n \mathrm{GE}=46 ; n \mathrm{GT}=59$ ) ont accepté de participer en signant le formulaire de consentement et ont complété le premier questionnaire. Ensuite, 90 ( $n \mathrm{GE}=41 ; n \mathrm{GT}=49$ ) étudiants ont complété les questionnaires de la mi-semestre (temps 2), puis 79 ( $n \mathrm{GE}=34$; $n \mathrm{GT}=45$ ) participants ont complété celui de la fin du semestre (temps 3). En tout 79 collégiens ( $36 \%$ d'hommes, tant dans le GE que dans le GT), ayant un âge moyen de 19 ans et répartis sur 4 groupes-classes, ont participé à tous les temps de mesure.

Cette organisation des GE et GT a contribué à contrôler les variables confondantes que peuvent être l'institution et l'enseignant, à défaut de pouvoir opter pour une procédure de randomisation ou d'insu. Toutefois, pour atteindre une taille d'échantillon intéressante dans la conduite des analyses comparatives, les deux GE devaient idéalement être fusionnés, de même que les deux GT. Pour contrôler l'impact d'un biais potentiel lié à l'appréciation de l'enseignant par les étudiants sur leur motivation envers le cours, ils ont été comparés sur la base de leurs scores à une échelle d'appréciation de l'enseignant, administrée à la fin du semestre ( $n$ présents $=78$ ). Les résultats à un test $t$ pour échantillons indépendants indiquent qu'il n'y a pas de différence $(t(77)=-, 63 ; p>, 05)$ dans l'appréciation de leur enseignant par les étudiants entre les deux enseignants. Ainsi, les deux groupes-classes de la condition expérimentale ont été fusionnés pour former le GE $(n=34)$ et il en a été de même pour les deux groupes-classes de la condition témoin $(n=45)$.

\subsection{Les conditions : expérimentale et témoin}

Dans le troisième cours d'ÉPS, l'étudiant doit planifier un Programme d'activités physiques (PAP) et l'exécuter en autonomie, tout en étant accompagné à distance par l'enseignant, par exemple grâce à une application mobile. Traditionnellement (condition témoin dans la présente étude), les étudiants font un test de course-navette de 20 mètres au premier cours du semestre, puis, sur la base de leur résultat, planifient leur PAP sous la supervision de 


\section{eJRIEPS 50 janvier 2022}

l'enseignant. L'objectif est d'arriver à atteindre les standards de performance révélant un niveau acceptable à excellent (Léger \& al., 2008) au test-navette à la fin du semestre. Logiquement, les étudiants prévoient beaucoup d'entrainement de course dans leur PAP puisqu'ils devront performer spécifiquement à cette activité standardisée 10 semaines plus tard.

Dans le cadre de la présente étude, la condition expérimentale implique principalement de permettre aux étudiants de choisir, dès le début du semestre, parmi quatre tests de la condition physique (course Cooper, course navette de 20 mètres, vélo Cooper, Step-test), celui par lequel sa condition cardiorespiratoire sera évaluée au début et à la fin du semestre. En effet, il n'apparaissait pas judicieux d'éliminer le test de la capacité cardiorespiratoire des cours d'EPS puisque de nombreux enseignants utilisateurs de ces tests ne semblent pas être prêts à faire ce changement de culture pédagogique. Le projet d'étude visait l'élaboration d'un mode d'évaluation plus contemporain, qui pourrait être adopté par le plus grand nombre d'enseignants.

\subsection{Instruments de mesure}

Un questionnaire de renseignements généraux a permis d'obtenir des données d'ordre général comme le genre, l'occupation à un emploi rémunéré, la pratique d'AP avec ses parents durant l'enfance ou le nombre d'heures hebdomadaire habituellement réservé à la pratique d'AP. Ces données ont permis d'explorer la similarité des groupes à comparer.

L'Échelle de la motivation en éducation physique (ÉMÉP, Cabot \& Bradette, 2021) a servi à mesurer la motivation des participants, ressentie envers le cours (les apprentissages et les évaluations qui y sont faits). La version prétest de l'ÉMÉP a été administrée lors du premier cours du semestre et la version posttest a été administrée lors du dernier cours du même semestre. Ce questionnaire est composé de 11 items de type Likert en 5 points allant de 1 (pas du tout en accord) à 5 (tout à fait en accord). Ces items représentent trois déterminants de la motivation: l'intérêt (4 items; p. ex. Je pense que je vais aimer assister à ce cours [prétest; $\lambda=, 89$ ] et J'ai aimé assister à ce cours [posttest; $\lambda=, 89$ ]), l'utilité (4 items; p. ex. Je crois que ce qu'on va apprendre dans ce cours d'éducation physique sera utile pour mon avenir [prétest; $\lambda=, 84$ ] et Je trouve que ce qu'on a appris dans ce cours d'éducation physique sera utile pour mon avenir [posttest; $\lambda=, 87$ ]) et la contrôlabilité (3 items; p. ex. Lors de ce cours d'éducation physique, je m'attends à pouvoir choisir la manière d'être évalué [prétest; $\lambda=, 80$ ] et Lors de ce cours d'éducation physique, j'ai pu 


\section{eJRIEPS 50 janvier 2022}

choisir la manière d'être évalué [posttest; $\lambda=, 80]$ ]. Les qualités métrologiques de l'instrument sont décrites dans [Cabot \& Bradette] (2021) et sont jugées très satisfaisantes.

Le sentiment de compétence à l'égard de la pratique d'AP a été mesuré par une échelle de type Likert, inspirée de celle de Losier \& al. (1993), et adaptée au contexte de la pratique d'AP. Elle est composée de quatre items (ex. : Je suis bon (bonne) pour faire de l'activité physique). Pour en explorer la validité, une analyse en composantes principales a été conduite sur ces items auprès de l'échantillon de la présente étude. Les résultats indiquent que les items saturent tous sur le même facteur et que les indices de saturation sont très satisfaisants (,85 à ,92). La fidélité de l'instrument a été estimée par le lambda-6 de Guttman (Bourque \& al., 2019) qui s'est aussi révélé satisfaisant $(\lambda=, 89)$.

La perception de la stratégie d'évaluation en EPS a été évaluée par une analyse thématique inductive des contenus des réponses fournies lors d'entrevues individuelles post-intervention. Malgré plusieurs rappels, onze étudiants (huit du GE et trois du GT) ont participé à cette entrevue semi-structurée, ayant duré entre 10 et 20 minutes selon le participant. Le canevas prévoyait cinq questions (précisées à la section 5.3) permettant d'estimer leur perception de la condition d'évaluation (expérimentale ou témoin) qu'ils avaient vécue et leur appréciation du cours.

\subsection{Procédures}

Lors du premier cours d'EPS du semestre d'automne 2019, les étudiants des quatre groupes-classes visés ont reçu l'information relative à l'étude, ont signé le formulaire de consentement et ont complété le Questionnaire de renseignements généraux, la version prétest de l'ÉMÉP et l'échelle du sentiment de compétence à l'égard de la pratique d'AP. Lors de la dernière semaine de cours, ils ont complété à nouveau cette dernière, ainsi que la version posttest de l'ÉMÉP. Au printemps 2020, la chercheuse principale a mené des entrevues téléphoniques avec les étudiants y ayant consenti.

Entre les deux cueillettes de données par questionnaires, la stratégie pédagogique s'est déroulée sur dix semaines. Concrètement, celle-ci comprend plusieurs tâches formatives en début de semestre et sommatives en fin de semestre. Premièrement, un test cardiorespiratoire formatif est effectué par l'étudiant. Deuxièmement, l'étudiant planifie et remet son PAP de manière formative à l'enseignant afin que ce dernier puisse orienter le travail par une rétroaction personnalisée. Troisièmement, l'étudiant réajuste son PAP et le met en pratique, hors cours, avec un accompagnement par l'enseignant à l'aide d'une application mobile permettant d'enregistrer et de démontrer l'intensité, le temps, et l'activité 


\section{eJRIEPS 50 janvier 2022}

réalisée. Quatrièmement, l'étudiant est invité à réfléchir à ses habitudes de vie et à appliquer une progression à son PAP au milieu du semestre. Cinquièmement, une reprise du test cardiorespiratoire en fin de semestre ainsi qu'une réflexion finale sur le processus effectué tout au long du semestre, visant à observer l'influence de la pratique d'AP sur son mode de vie et sa santé, sont effectués. Tous les participants ont vécu cette stratégie pédagogique, à l'exception du fait d'avoir le choix (GE) ou non (GT) du test cardiorespiratoire.

\subsection{Plan d'analyse}

Pour apprécier clairement la similarité des groupes à comparer, des tests d'hypothèse ont d'abord été calculés sur les données descriptives de cinq variables potentiellement confondantes : le genre, le fait d'avoir ou non un emploi rémunéré, le fait d'avoir pratiqué ou non de l'activité physique avec ses parents durant l'enfance, le nombre d'heures par semaine d'AP pratiquée hors cours, ainsi que l'intérêt ressenti pour l'exercice physique en général. Les données de ces cinq variables ont été obtenues avant le début de l'intervention. Dans un deuxième temps, les corrélations partielles ont été calculées entre les principales variables de l'étude, en contrôlant le groupe d'appartenance. Ensuite, des analyses de covariance ont été menées sur les données d'intérêt, d'utilité et de contrôlabilité, en considérant les mesures d'attentes comme covariables. Quatrièmement, une analyse de variance à mesures répétées a été conduite sur les données de sentiment de compétence à pratiquer de l'AP, mesurées au début et à la fin du semestre. Enfin, des analyses de contenu d'entrevues, menées après la fin du semestre d'intervention, ont permis de savoir si la condition d'évaluation (expérimentale ou témoin) avait bien été perçue par les participants.

\section{Résultats}

\subsection{Analyses descriptives}

Le GE et le GT ont été comparés sur la base des principales variables du Questionnaire de renseignements généraux pour en explorer la similarité avant la conduite des analyses principales. Les résultats sont présentés dans le tableau I et indiquent que les groupes étaient similaires au départ. 
eJRIEPS 50 janvier 2022

Tableau I : Données descriptives et résultats aux tests comparant les groupes au départ

\begin{tabular}{|c|c|c|c|}
\hline Variables & GE $(n=34)$ & GT $(n=45)$ & tests \\
\hline Genre & $9 \mathrm{~h} / 25 \mathrm{f}$ & $12 \mathrm{~h} / 33 \mathrm{f}$ & $K h i^{2}=, 00 \quad(p>, 05)$ \\
\hline Emploi rémunéré & 24 oui & 32 oui & $K h i^{2}=, 00 \quad(p>, 05)$ \\
\hline $\begin{array}{l}\text { AP régulière avec parents } \\
\text { durant l'enfance }\end{array}$ & 22 oui & 28 oui & $K h i^{2}=2,39(p>, 05)$ \\
\hline $\begin{array}{l}\text { Nombre d'heures/sem } \\
\text { d'AP hors cours }\end{array}$ & $\begin{array}{c}2,62 \\
(e ́-t=2,30)\end{array}$ & $\begin{array}{c}3,33 \\
(e-t=2,68) \\
\end{array}$ & $F=1,19 \quad(p>, 05)$ \\
\hline $\begin{array}{l}\text { Intérêt pour l'exercice } \\
\text { physique en général } \\
\text { (Likert en } 4 \text { points) }\end{array}$ & $\begin{array}{c}2,24 \\
(e ́-t=, 74)\end{array}$ & $\begin{array}{c}2,20 \\
(e ́-t=, 81)\end{array}$ & $F=, 78 \quad(p>, 05)$ \\
\hline
\end{tabular}

Ensuite, les corrélations partielles ont été calculées entre les principales variables à l'étude (tableau II) en contrôlant le groupe d'appartenance (GE ou GT). C'est-à-dire que l'effet potentiel du groupe a été maintenu constant durant le calcul pour en évacuer la part de variance commune aux variables corrélées. On observe que les variables motivationnelles à l'égard du cours, mesurées au même temps de mesure (cellules ombragées), sont corrélées ensemble, tel que la littérature permet de s'y attendre. De plus, chaque concept motivationnel est corrélé avec lui-même entre les deux temps de mesure. On constate aussi que l'intérêt ressenti à l'égard du cours est très fortement lié à toutes les variables motivationnelles mesurées à la fin du semestre (cellules en gras), tant celles qui concernent le cours (utilité et contrôlabilité) que celle qui concerne la pratique d'activités physiques (sentiment de compétence). 
eJRIEPS 50 janvier 2022

Tableau II: Corrélations partielles entre les principales variables à l'étude (variable contrôlée : groupe d'appartenance - GE ou GT)

\begin{tabular}{|c|c|c|c|c|c|c|c|c|}
\hline & 1 & 2 & 3 & 4 & 5 & 6 & 7 & 8 \\
\hline \multicolumn{9}{|l|}{$\begin{array}{l}\text { 1. Attentes } \\
\text { d'intérêt cours }\end{array}$} \\
\hline $\begin{array}{l}\text { 2. Attentes } \\
\text { d'utilité cours }\end{array}$ &, $54^{* \star *}$ & & & & & & & \\
\hline $\begin{array}{l}\text { 3. Attentes de } \\
\text { control. cours }\end{array}$ &, $30^{\star *}$ &, $31^{\star *}$ & & & & & & \\
\hline 4. Intérêt cours & $46^{* * *}$ & ,23* & ,11 & & & & & \\
\hline 5. Utilité cours & ,28* &, $55^{\star * *}$ & ,21 &, $58^{\star \star \star}$ & & & & \\
\hline $\begin{array}{l}\text { 6. Contrôlabilité } \\
\text { cours }\end{array}$ &, $29^{* \star}$ & ,20 &, $46^{* * *}$ &, $38^{* * *}$ &, $28^{*}$ & & & \\
\hline $\begin{array}{l}\text { 7. Sentiment de } \\
\text { comp. AP (pré) }\end{array}$ &, $43^{* * *}$ & ,19 &, $42^{* * *}$ &, $26^{*}$ & ,07 & ,13 & & \\
\hline $\begin{array}{l}\text { 8. Sentiments de } \\
\text { comp. AP (post) }\end{array}$ &, $37^{* * *}$ & , 16 & ,22 &, $52^{\star \star \star}$ &, $30^{* *}$ &, $25^{\star}$ &, $69^{* \star *}$ & \\
\hline$\overline{\bar{x}}$ & 3,72 & 3,80 & 3,08 & 3,82 & 4,04 & 3,28 & 3,30 & 3,46 \\
\hline é-t & ,79 & ,69 & 1,00 & ,84 & ,84 & 1,04 & 94 & ,92 \\
\hline
\end{tabular}

Notes. $n=79 ;{ }^{*} p<, 05 ;{ }^{* *} p<, 01 ;{ }^{* * *} p<, 001$.

\subsection{Analyses comparatives}

En ce qui concerne les données prétest et posttest découlant de l'ÉMÉP, des analyses de covariance (ANCOVA) ont été menées en considérant les mesures prétest comme covariables aux mesures posttest. Les résultats sont exposés dans le tableau III. On y constate des différences fortement significatives entre les groupes quant à l'intérêt et à la contrôlabilité à l'égard du cours, ainsi que des tailles d'effet modestes (mesurées par $\eta^{2}$ p). 
eJRIEPS 50 janvier 2022

Tableau III : Scores moyens, (écarts-types) et résultats des ANCOVA opérées sur les données de motivation à l'égard du cours d'EPS

\begin{tabular}{|c|c|c|c|}
\hline & GE $(n=34)$ & GT $(n=45)$ & $\begin{array}{c}F(1,79) \\
\eta^{2} p\end{array}$ \\
\hline \multicolumn{4}{|l|}{ Intérêt } \\
\hline $\begin{array}{l}\text { Covariable : attentes d'intérêt } \\
\text { pour le cours d'EPS }\end{array}$ & $3,69(, 78)$ & $3,74(, 82)$ & $\begin{array}{c}20,41^{\star \star \star} \\
, 21\end{array}$ \\
\hline Groupes & $4,14(, 57)^{1}$ & $3,57(, 93)^{1}$ & $\begin{array}{c}13,51^{\star \star \star} \\
, 15\end{array}$ \\
\hline \multicolumn{4}{|l|}{ Utilité } \\
\hline $\begin{array}{l}\text { Covariable : attentes d'utilité } \\
\text { relative au cours d'EPS }\end{array}$ & $3,76(, 65)$ & $3,84(, 74)$ & $\begin{array}{c}33,40^{* * *} \\
, 31\end{array}$ \\
\hline Groupes & $4,10(, 78)^{2}$ & $4,00(, 88)^{2}$ & $\begin{array}{l}, 88 \\
, 01\end{array}$ \\
\hline \multicolumn{4}{|l|}{ Contrôlabilité } \\
\hline $\begin{array}{l}\text { Covariable : attentes de } \\
\text { contrôlabilité sur cours d'EPS }\end{array}$ & $3,14(1,05)$ & $3,04(, 98)$ & $\begin{array}{c}20,10^{\star \star *} \\
, 21\end{array}$ \\
\hline Groupes & $3,80(, 78)^{3}$ & $2,89(1,04)^{3}$ & $\begin{array}{c}20,78^{\star \star *} \\
, 22\end{array}$ \\
\hline
\end{tabular}

${ }^{\star \star \star} p<, 001$.

${ }^{1}$ Variable dépendante: Intérêt pour le cours d'EPS tel que ressenti en fin de semestre.

${ }^{2}$ Variable dépendante: Utilité pour le cours d'EPS telle que ressentie en fin de semestre.

${ }^{3}$ Variable dépendante: Contrôlabilité pour le cours d'EPS telle que ressentie en fin de semestre.

Comme les items mesurant le sentiment de compétence sont les mêmes, avant et après l'intervention, une analyse de variance (ANOVA) à mesures répétées a été opérée sur ces données. Les résultats sont détaillés au tableau IV. On y constate un changement lié au temps sans effet de groupe, c'est-à-dire tant chez le GE que chez le GT.

Tableau IV : Scores moyens, (écarts-types) et résultats de l'ANOVA à mesures répétées, quant au sentiment de compétence à pratiquer de l'AP

\begin{tabular}{lccccccc}
\hline Variable & \multicolumn{2}{c}{ GE $(\mathbf{n}=\mathbf{3 4})$} & \multicolumn{2}{c}{ GT $(\mathbf{n}=\mathbf{4 5})$} & \multicolumn{2}{c}{$\begin{array}{c}\text { Valeurs de } \boldsymbol{F} \\
\text { Taille d'effet }\left(\mathbf{n}^{2} \mathbf{p}\right)\end{array}$} \\
\hline & Temps 1 & Temps 2 & Temps 1 & Temps 2 & Temps & Groupe & $\begin{array}{c}\text { Temps X } \\
\text { Groupe }\end{array}$ \\
\hline Sentiment de & 3,12 & 3,44 & 3,39 & 3,52 & $4,39^{*}$ & 1,35 & 1,41 \\
compétence AP & $(1,01)$ & $(, 87)$ & $(, 81)$ & $(1,00)$ &, 05 &, 02 &, 02 \\
\hline
\end{tabular}

${ }^{*} p<, 05$. 


\section{eJRIEPS 50 janvier 2022}

\subsection{Analyses des contenus des entrevues}

Une synthèse des contenus découlant des entrevues est présentée dans le tableau V. La première question, plus générale, servait de question d'amorce Tous les participants ( $n=11)$ ont répondu par des commentaires positifs à cette question. De plus, trois étudiants du GE ont spontanément exprimé qu'ils avaient aimé pouvoir choisir leur test final. La deuxième question servait d'assise préalable à un des objectifs de l'entrevue, qui était d'amener les étudiants du GE à comparer les deux conditions. Les réponses à la troisième question ont permis de constater que la condition expérimentale a bien été perçue par les participants du GE.

La quatrième question, dont la formulation était adaptée au groupe d'appartenance, invitait les répondants à faire l'exercice de comparer les deux conditions (test final imposé ou test final au choix). 
eJRIEPS 50 janvier 2022

Tableau V : Synthèse des contenus découlant de l'entrevue, en fonction des questions posées

\begin{tabular}{|c|c|c|}
\hline Questions d'entrevue & Synthèses & Exemples de verbatim \\
\hline $\begin{array}{l}\text { Q.1 Comment s'est passé ton cours } \\
\text { d'éducation physique à la dernière } \\
\text { session? }\end{array}$ & $\begin{array}{l}\text { Satisfaction générale } \\
(n=11)\end{array}$ & $\begin{array}{l}\text { J'ai aimé les entrainements. } \\
\text { Ça s'est bien passé Difficile, mais j'ai apprécié. }\end{array}$ \\
\hline $\begin{array}{l}\text { Q.2 Est-ce que tu as déjà eu un cours } \\
\text { d'éducation physique dans lequel tu as } \\
\text { dû faire un test navette obligatoire à la } \\
\text { fin du cours? }\end{array}$ & $\begin{array}{l}\text { Réponse Oui } \\
(n=11)\end{array}$ & $\begin{array}{l}\text { Je ne l'aime vraiment pas ce test, j'aimerais le faire à mon intensité. Je trouve } \\
\text { cela plate et long. } \\
\text { Le test est difficile, la première fois j'ai vomi. } \\
\text { J'aime ce test, car c'est comme un défi, cela me motive. }\end{array}$ \\
\hline $\begin{array}{l}\text { Q.3 Pour GE (valider la perception de la } \\
\text { condition expérimentale) : À la dernière } \\
\text { session, te souviens-tu que tu as pu } \\
\text { choisir ton test cardiorespiratoire de fin } \\
\text { de session? (Comment ça s'est passé? } \\
\text { Appréciation/satisfaction? Quel test as-tu } \\
\text { choisi? Pour quelles raisons?) }\end{array}$ & $\begin{array}{l}\text { Réponse « oui » } \\
(n=8) \\
\text { Tests choisis : } \\
\text { Cooper de course : } \\
n=4 \\
\text { Step-test : } n=1 \\
\text { Test vélo : } n=2 \\
\text { Course navette: } \\
n=1\end{array}$ & $\begin{array}{l}\text { Quand je l'ai fait, ça s'est bien passé. J'ai choisi le test Cooper car je suis } \\
\text { habitué en endurance, je fais souvent du vélo et de la marche rapide hors } \\
\text { cours. } \\
\text { J'avais le choix, j'avais le goût d'essayer un nouveau test. } \\
\text { J'ai pris le Step-test, car je suis très paresseux dans la vie et que je n'aime pas } \\
\text { courir et faire des tests physiques. } \\
\text { Parce que j'ai vu que dans le cardio vélo, mon corps s'adaptait plus facilement } \\
\text { avec cela. J'étais un peu plus capable de trouver des moyens pour faire } \\
\text { augmenter ma performance. } \\
\text { Parce que c'est quelque chose que j'aime vraiment, le vélo. }\end{array}$ \\
\hline $\begin{array}{l}\text { Q.4. Pour le GE : comparer les deux } \\
\text { conditions. } \\
\text { Selon toi, le fait d'avoir pu choisir ton } \\
\text { test lors de ton dernier cours a-t-il pu } \\
\text { avoir un effet sur ta motivation à mettre } \\
\text { en application ton PAP régulièrement, }\end{array}$ & $\begin{array}{l}\text { GE }(n=8) \\
\text { Oui : } n=6 \\
\text { Non : } n=2\end{array}$ & $\begin{array}{l}\text { Non, parce que je suis déjà très actif dans la vie et que j'aurais fait les mêmes } \\
\text { activités physiques en ayant n'importe quel test d'évaluation, mais si j'étais } \\
\text { moins actif cela m'aurait vraiment plus motivé. }\end{array}$ \\
\hline
\end{tabular}




\section{pour performer à ce test en fin de} session?

Pour le GT : Aurais-tu préféré avoir le choix parmi différents tests cardiorespiratoires? Par exemple course, vélo, step. (Expliquer la réponse. Quel test crois-tu que tu aurais choisi? Pourquoi?)

Q.5 Si tu penses à ces deux conditions d'évaluation du cours d'éducation physique (test imposé ou test choisi), crois-tu que cela peut influencer ta tendance à pratiquer de l'activité physique à long terme? GT $(n=3)$
Oui $=(n=1)$
N'importe quel choix de test cardiovasculaire pour moi c'est sérieux et très difficile. Non $=(n=1)$

Indifférent $=(n=1)$

Oui $=(n=9)$

Je pense que si on donne le choix d'activité physique cela encourage différentes manières de s'entrainer.

Oui. Le cours m'a donné le goût de pratiquer de l'activité physique (participant du GE - test choisi).

Si tu as le choix de choisir quelque chose que tu peux aimer, je pense que tu peux plus facilement l'apprécier en dehors du cours.

Je pense que oui, car ce serait moins démoralisant sachant qu'en vélo je pourrais mieux performer qu'au test navette.

Non $=(n=2)$
Cela ne fera pas changer ma pratique à long terme.

Non je ne pense pas que ça a un lien. 


\section{eJRIEPS 50 janvier 2022}

Pour la dernière question, il était d'abord demandé aux participants de réfléchir aux deux conditions d'évaluation possibles (test imposé ou test au choix), puis d'exprimer son point de vue sur l'influence que cela peut avoir sur la tendance à pratiquer de l'AP à long terme. Six des huit répondants du GE ont affirmé qu'un choix de test influençait positivement leur tendance à pratiquer de l'AP à long terme. La récurrence de ce commentaire corrobore le potentiel du respect des intérêts en matière d'AP comme bras de levier dans l'adoption d'un mode de vie sain et actif hors cours. Les deux autres participants du GE ont mentionné que le choix d'un test cardiorespiratoire n'avait pas d'influence sur leur niveau de pratique d'AP à long terme. Cependant, l'un d'entre eux a précisé que le fait de choisir un test amène une variété et une découverte de tests et que cela diminue la pression de performance et de comparaison sociale, vécues lors du test de course navette, tout en augmentant le sentiment de compétence. Quant à l'autre répondant, il mentionne que de se faire imposer un test permet de découvrir quelque chose qu'on n'aurait pas choisi initialement et que l'on doive s'y adapter et se surpasser pour devenir meilleur.

\section{Discussion et limites}

Les résultats obtenus justifient qu'une évaluation plus approfondie de son impact soit conduite, impliquant plus de variables, notamment un suivi longitudinal de la pratique d'AP. De plus, un échantillon incluant un grand nombre de groupes-classes permettrait de limiter les effets du biais potentiellement induit par l'appréciation de l'enseignant par les étudiants, bien qu'il demeure pertinent d'avoir le souci de contrôler ce biais, comme dans la présente étude. De surcroît, il serait intéressant d'élargir le spectre des choix, par exemple en ajoutant un test de nage dans les établissements où les installations le permettent.

Le résultat principal est assurément celui qui concerne la contrôlabilité rapportée par le GE de façon plus marquée que celle exprimée par le GT. Comme l'intervention pédagogique visait précisément à donner plus de contrôle aux étudiants pour combler leur besoin d'autonomie, on s'attendait à trouver une différence claire comparée au GT sur cette variable, ce qui est le cas.

De plus, sur un plan intra-individuel, les résultats indiquent que la contrôlabilité vécue par les étudiants du GE a été plus élevée que ce à quoi ils s'attendaient au départ. Les attentes de contrôlabilité exprimées en début de semestre sont possiblement le reflet des expériences passées à être évalué à travers un cadre traditionnellement plus rigide et imposé. Bref, ces résultats comparatifs, tant intra qu'inter-groupes, confirment que le fait 


\section{eJRIEPS 50 janvier 2022}

d'offrir des choix aux étudiants pour qu'ils perçoivent avoir plus de contrôle sur le déroulement du cours est une stratégie qui atteint sa cible.

Un autre résultat important concerne la différence marquée d'intérêt ressenti à l'égard du cours d'EPS, à l'avantage du GE. Ce résultat ainsi que la littérature laissent croire que le contrôle offert à l'étudiant suscite son intérêt envers le cours d'EPS. En effet, certains auteurs (Patall \& Yang Hooper, 2019; Patall, 2013) expliquent l'intérêt et la contrôlabilité par l'exercice de choix comme étant des variables interreliées. En effet, pour être motivant, le fait de pouvoir choisir doit permettre à l'étudiant d'exprimer ses préférences et d'agir sur la base de ses intérêts (Patall \& Yang Hooper, 2019). Au contraire, lorsque les modalités sur lesquelles l'étudiant peut exercer du contrôle ne sont pas alignées avec ses intérêts, l'exercice de ce contrôle est perçu comme non pertinent. Conséquemment, si les modalités sur lesquelles l'étudiant peut exercer du contrôle dans la manière d'être évalué en EPS lui permettent de choisir des activités qui l'intéressent, cette contrôlabilité est signifiante pour lui. L'intérêt pourrait donc avoir préséance sur la contrôlabilité, cette dernière étant un instrument permettant l'atteinte de l'objet d'intérêt. Ce type d'interprétation concorde aussi avec les vocables de certains participants aux entrevues, comme " choisir quelque chose que l'on aime, c'est plus motivant ». Suivant cette veine, si les intérêts préexistants des étudiants ont pu être respectés à travers l'exercice du choix d'AP, et considérant que la majorité des répondants du GE a affirmé que cela avait une influence positive sur leur engagement à pratiquer de l'AP quelques mois après la fin du cours, ces résultats contribuent à affirmer le pouvoir de l'intérêt sur l'engagement et la persévérance. Ces observations sont d'autant plus en cohérence avec les travaux de Roure \& Pasco (2020) énonçant que de susciter le plaisir instantané de bouger ainsi que l'intérêt en situation d'AP seraient un gage de stratégie pédagogique efficace.

Quant à la variable d'utilité, une différence intra-individuelle a été constatée dans les deux groupes, indiquant que les étudiants ont tendance à juger les activités faites dans leur dernier cours d'EPS comme plus utiles que ce à quoi ils s'attendaient au départ. Ce résultat est encourageant considérant que ces cours sont la dernière opportunité de fournir un accompagnement pédagogique aux jeunes adultes dans la prise en charge de leur pratique d'AP. De plus, les scores très élevés (près de la borne supérieure de l'échelle) d'utilité dans les deux groupes pourraient indiquer que les jeunes adultes considèrent l'AP utile en soi, ce qui pourrait expliquer l'absence de différence entre les groupes. En effet, les étudiants du postsecondaire ont passé plus de dix ans à réussir des cours d'EPS depuis l'école primaire. Ce résultat pourrait donc s'expliquer par la reconnaissance, chez les étudiants au 


\section{eJRIEPS 50 janvier 2022}

postsecondaire, de l'importance de l'AP dans les saines habitudes de vie et la santé. Sur le plan conceptuel, ce résultat contribue à renforcer la pertinence de distinguer l'intérêt et l'utilité dans l'étude de la dynamique motivationnelle (Cabot, 2017).

Finalement, le sentiment de compétence des étudiants envers la pratique d'activités physiques a augmenté durant le semestre sans se distinguer en fonction de l'appartenance au GE ou au GT. Ceci est, somme toute, une bonne nouvelle, car il est possible de supposer qu'une pratique d'AP récurrente, par le biais des cours d'EPS dans les deux groupes, explique l'augmentation similaire du sentiment de compétence spécifique à la pratique d'AP. Logiquement, l'étudiant invité à participer à des AP dans le cadre du cours d'EPS s'améliore, vit des expériences positives ainsi que des réussites pédagogiques qui forgent son sentiment de compétence à faire de l'AP. D'autre part, proposer des activités physiques en cohérence avec les intérêts des étudiants vise ultimement la pérennisation de la pratique d'AP. Le but premier étant de faire bouger ces jeunes adultes dans le respect de leurs intérêts, en visant le plaisir pour que d'eux-mêmes ils perpétuent ensuite ce mode de vie sain et actif après la fin de leurs cours d'EPS. À cet effet, il apparait recommandé de faire vivre aux étudiants des expériences pédagogiques stimulantes, en respectant leur besoin d'autonomie, et ce, dans le plaisir de bouger, tel que le préconisent aussi d'autres équipes de chercheurs (Lemoyne \& Girard, 2018; Roure, Lentillon-Kaestner \& al., 2019; Roure, Méard \& al., 2019). Toutefois, la principale limite contenant la portée de cette étude est la taille de l'échantillon. Comme il s'agissait de faire une première exploration en contexte réel de la stratégie d'évaluation élaborée par Bradette (2020), peu de groupes-classes ont été sollicités.

\section{Conclusion}

Un désengagement envers la pratique de l'AP ainsi qu'une baisse de la motivation pour le cours d'EPS sont observés chez les adolescents et jeunes adultes du postsecondaire. À cette étape du développement, l'étudiant désire plus de liberté et de responsabilités dans sa vie et cela se perçoit aussi dans son besoin d'autonomie à travers ses cours. Cette étude visait à tester les impacts d'une stratégie d'évaluation, permettant un choix de test cardiorespiratoire en fonction des préférences sportives de l'étudiant, sur sa motivation envers son cours d'EPS. Les résultats de l'étude laissent croire que la contrôlabilité offerte rejoint les besoins d'autonomie des étudiants et cela se transpose dans une augmentation de l'intérêt ressenti envers leur dernier cours d'EPS du cheminement scolaire. Ces résultats 


\section{eJRIEPS 50 janvier 2022}

doivent encourager les enseignants à utiliser des stratégies évaluatives innovantes et tenant compte des besoins des étudiants.

L'efficacité de cette stratégie d'évaluation devrait être étudiée davantage. Par exemple, il serait fort pertinent de transférer cette stratégie d'évaluation dans diverses disciplines du troisième cours d'EPS aux études postsecondaires. De plus, de grands échantillons permettraient de raffiner les analyses en tenant compte de diverses variables, comme le genre et les habitudes sportives. Ainsi, l'on pourrait observer l'impact d'une telle stratégie d'évaluation en fonction des capacités et intérêts hétérogènes des étudiants au postsecondaire. Enfin, découlant du contexte de pandémie, il serait intéressant d'étudier l'apport d'une telle stratégie d'évaluation sur la motivation en EPS, qui doit parfois être enseignée à distance, et la prise en charge autonome d'AP par les étudiants.

Cette étude s'est terminée en contexte de pandémie. Par exemple, les entrevues ont eu lieu en mars 2020, durant le premier confinement dû à la Covid-19. À l'origine, deux mesures de suivi de la pratique de l'AP post-cours étaient prévues au printemps et à l'automne 2020. Ces deux cueillettes de données ont eu lieu durant les deux premières vagues de la pandémie. Cette situation a invalidé les données recueillies. En effet, étant donné le contexte, la chercheuse principale a demandé aux participants de s'exprimer sur l'effet du contexte pandémique sur leur pratique d'AP. La majorité des répondants ont rapporté une influence très négative du confinement prolongé sur leur motivation à bouger. Ces réponses sont en harmonie avec les données de recherches qui commencent à émerger indiquant déjà des conséquences négatives des mesures de confinement sur la pratique d'activités physiques. Néanmoins, une des questions à l'origine de ces mesures de suivi de l'AP était de savoir si une augmentation de la motivation envers le dernier cours d'EPS dans un contexte d'autonomie aurait pu avoir une influence positive à long terme par l'intégration de l'AP au mode de vie et ainsi représenter un point de bascule entre l'intérêt en situation et l'intérêt individuel (Chen \& Wang, 2017). Suivant cette idée, de telles mesures longitudinales devraient être intégrées à un projet de réplication de cette étude, en contexte non pandémique, idéalement auprès d'un large échantillon, afin de constater réellement les impacts d'une telle stratégie d'évaluation innovante sur la pratique pérenne de l'AP.

\section{Bibliographie}

Cour Supérieur [QCCS]. (2012). Dallaire-Turmel c. Cégep de Lévis-Lauzon, 2012 QCCS 647 (CanLII). http://canlii.ca/t/fab6f 
eJRIEPS 50 janvier 2022

Alexander, P. A. \& Jetton, T. L. (1996). The role of importance and interest in the processing of text. Educational Psychology Review, 8(1), 89-121. https://doi.org/10.1007/BF01761832

Ames, C. (1992). Classrooms: Goals, Structures, and Student Motivation. Journal of Educational Psychology, 84(3), 261-271. https://doi.org/10.1037/0022$\underline{0663.84 .3 .261}$

Bates, A. W. (2015). Teaching in a Digital Age: Guidelines for Designing Teaching and Learning for a Digital Age. BCCampus.

Baril, G., Paquette, M.-C., Ouimet, A.-M., Laguë, J., Marquis, M., Séguin-Tremblay, R. \& Nolin, B. (2014). Ados 12-14: les dimensions socioculturelles des pratiques alimentaires et d'activité physique des adolescents. Institut National de santé publique. Québec. https://www.inspq.qc.ca/pdf/publications/1773 Ados1214DimSocioPratAlimActPhyAdo.pdf

Bélanger, D.-C. \& Tremblay, K. (2012). Portrait actualisé des croyances et des pratiques en évaluation des apprentissages au collégial. Rapport regroupement des collèges Performa. Collège de Maisonneuve.

Bourque, J., Doucet, D., LeBlanc, J., Dupuis, J. \& Nadeau, J. (2019). L'alpha de Cronbach est l'un des pires estimateurs de la consistance interne : une étude de simulation. Revue des sciences de l'éducation, 45(2), 78-99. https://doi.org/10.7202/1067534ar

Brand, O. (2015). Exploration des liens entre morphologie et motivation au cours d'éducation physique: comment optimiser l'intégration des élèves en surpoids dans les cours d'éducation physique au cycle d'orientation et développer leur envie de pratiquer des exercices physiques. Mémoire professionnel. Haute école pédagogique du Valais, Genève.

Bradette, A. (2020). Conception et évaluation d'une évaluation terminale motivant les collégiennes et les collégiens à la pratique pérenne d'activités physiques (Essai de Maîtrise). Université de

Sherbrooke. https://savoirs.usherbrooke.ca/handle/11143/17341

Bradette, A. \& Cabot, I. (2019). Comment susciter la motivation des étudiants en éducation physique de l'ensemble 3 ? Revue Propulsion, 32(1), 30-34.

Cabot, I. (2017). Le potentiel d'influence de l'intérêt scolaire dans la motivation des collégiens en difficulté. Article de conférence. Journée de la recherche sur la motivation au collégial, $2^{e}$ édition. Congrès de l'Acfas, Montréal. 
eJRIEPS 50 janvier 2022

https://eduq.info/xmlui/bitstream/handle/11515/34809/cabot-potentiel-influenceinteret-scolaire-motivation-collegiens-en-difficulte-article-acfas-2017.pdf

Cabot, I. \& Bradette, A. (2021, soumis). Élaboration et validation initiale de l'Échelle de la motivation en éducation physique (ÉMÉP) auprès d'étudiants au postsecondaire.

Cale, L., Harris, J., \& Chen, M.-H. (2012). Monitoring health, activity and fitness in physical education: Its current and future state of health. Sport, Education and Society, 19(4), 376-397. https://doi.org/10.1080/13573322.2012.681298

Carroll, B. (1994). Assessment in Physical Education: A Teacher's Guide to the Issues. Falmer Press.

Chen, A. (2001). A Theoretical Conceptualization for Motivation Research in Physical Education: An Integrated Perspective. Quest, 53(1), 35-58. https://doi.org/10.1080/00336297.2001.10491729

Chen, A., \& Darst, P. W. (2001). Situational Interest in Physical Education : A Function of Learning Task Design. 16. https://doi.org/10.1080/02701367.2001.10608945

Chen, A. \& Hancock, G. R. (2006). Conceptualizing a Theoretical Model for SchoolCentered Adolescent Physical Activity Intervention Research. Quest, 58(3), 355-376. https://doi.org/10.1080/00336297.2006.10491887

Chen, A. \& Wang, Y. (2017). The Role of Interest in Physical Education: A Review of Research Evidence. Journal of Teaching in Physical Education, 36, 313-322. https://doi.org/10.1123/itpe.2017-0033

Chen, S., Sun, H., Zhu, X. \& Chen, A. (2014). Relationship Between Motivation and Learning in Physical Education and After-School Physical Activity. Research Quarterly for Exercise and Sport, 85(4), 468-477. https://doi.org/10.1080/02701367.2014.961054

Chiasson, L. (2004). Sentiment d'efficacité personnelle, habitudes de vie et niveau de condition physique. Rapport de recherche PAREA.

https://cdc.qc.ca/parea/729790 chiasson Levis lauzon PAREA 2006.pdf

Chiasson, L., Lapointe, G. \& Leblanc, G. (2006). Le sentiment d'efficacité personnelle par rapport à certaines habitudes de vie. Rapport de recherche PAREA. https://cdc.qc.ca/parea/786491 chiasson Levis lauzon article PAREA 2006.pdf

Dubeau, A., Frenay, M. \& Samson, G. (2015). L'utilité perçue de la tâche: Présentation du concept et état de la recherche. Canadian Journal of Education, 38, 1-23. https://journals.sfu.ca/cje/index.php/cje-rce/article/view/1757 


\section{eJRIEPS 50 janvier 2022}

Evans, M. \& Boucher, A. (2015). Optimizing the Power of Choice: Supporting Student Autonomy to Foster Motivation and Engagement in Learning. Mind, Brain, and Education, 9(2), 87-91. https://doi.org/10.1111/mbe.12073

Fredricks, J. A., Blumenfeld, P. C. \& Paris, A. H. (2004). School Engagement: Potentiel of the Concept, State of the Evidence. Review of Educational Research, 74(1), 59-109. https://doi.org/10.3102/00346543074001059

Gadais, T. (2015). Les stratégies d'intervention pour aider les jeunes à gérer leur pratique d'activité physique. Une revue de la littérature. Staps, 109(3), 57-77. https://doi.org/10.3917/sta.109.0057

Gaudreault, K., R. Richards, K. A. \& Woods, A. (2016). Understanding the perceived mattering of physical education teachers. Sport, Education and Society, 1-13. https://doi.org/10.1080/13573322.2016.1271317

Girard, S. (2016). Climat motivationnel en éducation physique et besoins psychologiques: impact sur les buts d'accomplissement, les attitudes et les habitudes des élèves [Université de

Montréal].

https://papyrus.bib.umontreal.ca/xmlui/handle/1866/15903

Grandchamp, A., Méard, J. \& Quin, G. (2018). Les pratiques évaluatives en éducation physique: État des lieux et évolution au cours des 20 dernières années. https://orfee.hepl.ch/handle/20.500.12162/1345

Green, K. (2004). Physical education, lifelong participation and 'the couch potato society'. Physical Education et Sport Pedagogy, 9, 73-86. https://doi.org/10.1080/1740898042000208133

Hidi, S. \& Renninger, K. A. (2006). The Four-Phase Model of Interest Development. Educational Psychologist, $41(2)$, 111-127. https://doi.org/10.1207/s15326985ep4102 4

Howe, R. \& Ménard, L. (1993). Croyances et pratiques en évaluation des apprentissages: Étude des croyances et des pratiques des enseignants des cégeps à l'égard de l'évaluation des apprentissages. Collège Montmorency. http://www.cdc.qc.ca/parea/702242-howe-menard-croyances-pratiquesevaluation-montmorency-PAREA-1993.pdf

Hulleman, C. S., Kosovich, J., Barron, K. E., \& Daniel, D. (2017). Making Connections: Replicating and Extending the Utility Value Intervention in the Classroom. Journal of Educational Psychology, 109(3), 387- 404. http://dx.doi.org/10.1037/edu0000146 


\section{eJRIEPS 50 janvier 2022}

Institut de la statistique du Québec (2020). Population de 15 ans et plus, selon le plus haut certificat, diplôme ou grade obtenu, sexes réunis, provins, territoires et Canada, 2016. Institut de la statistique du Québec. Consulté le 9 avril 2021, à l'adresse : https://statistique.quebec.ca/fr/document/niveau-de-scolarite-et-domaine-detudesen-2016-selon-le-sexe-le-groupe-dage-et-la-regionadministrative/tableau/niv sco 2016\#tri tertr $=24$

Jaakkola, T., Saakslahti, A., Yli-piipari, S., Manninen, M., Watt, A. P., \& Liukkonen, J. (2013). Student Motivation Associated With Fitness Testing in the Physical Education Context. Journal of Teaching in Physical Education, 32, 270-286.

Katz, I. \& Assor, A. (2007). When Choice Motivates and When It Does Not. Educational Psychology Review, 19, 429-442. https://doi.org/10.1007/s10648-006-9027-y

Keating, X., Liu, X., Stephenson, R., Guan, J., \& Hodges, M. (2020). Student health-related fitness testing in school-based physical education: Strategies for student self-testing using technology. European Physical Education Review, 26(2), 552-570. https://doi.org/10.1177/1356336X19874818

Kino-Québec. (2020). Pour une population québécoise physiquement active: des recommandations. Ministère de l'Éducation et de l'Enseignement supérieur, Gouvernement du

Québec.

http://www.education.gouv.qc.ca/fileadmin/site web/documents/loisirsport/KINO P opulation physiquement active.pdf

Kino-Québec. (2014). L'activité physique et sportive des adolescentes: bilan, perspectives et pistes

d'action.

http://www.education.gouv.qc.ca/fileadmin/site web/documents/loisirsport/SLS sport bilan adolescentes FR.pdf

Kino-Québec (2012). Opération ados: des activités qui répondent à leurs goûts. http://www.kino-quebec.qc.ca/publications/operationados.pdf

Kirk, D. (2005). Physical education, youth sport and lifelong participation: The importance of early learning experiences. European Physical Education Review, 11. https://doi.org/10.1177/1356336X05056649

Kirk, D. (2013). Educational Value and Models-Based Practice in Physical Education. Educational Philosophy and Theory, 45, 973-986. https://doi.org/10.1080/00131857.2013.785352 


\section{eJRIEPS 50 janvier 2022}

Lalime, F. (2016). Élaboration d'une stratégie d'évaluation visant le développement de la compétence "activité physique et autonomie » du cours d'éducation physique de l'ensemble 3 au collégial. [Essai de Maîtrise]. Université Laval.

Léger, L. et collab. (2008). Trousse d'évaluation de l'aptitude physique : Manuel d'instruction pour l'utilisation des tests. Société canadienne de physiologie de l'exercice, Ottawa. Lemoyne, J. (2012). Éducation Physique: Vers l'adoption d'un mode de vie actif? Rapport de recherche PAREA, 192. https://eduq.info/xmlui/bitstream/handle/11515/29807/788349-lemoyne-educationphysique-collegial-shawinigan-PAREA-2012.pdf?sequence $=1$ \&isAllowed $=y$

Lemoyne, J. \& Girard, S. (2019). Motiver les étudiants du collégial à bouger : regard sur la contribution des cours d'éducation physique. Article de conférence. Journée de la recherche sur la motivation au collégial, $2^{e}$ édition. Congrès de l'Acfas, Montréal. https://eduq.info/xmlui/bitstream/handle/11515/37729/lemoyne-girard-acfas2019.pdf? sequence $=2 \&$ is Allowed $=y$

Lemoyne, J., \& Girard, S. (2018). Activité physique, estime de soi et condition physique: étude longitudinale d'une cohorte d'étudiants québécois. Staps, (2), 99-115.

Lemoyne, J. \& Valois, P. (2012). Prédicteurs de la pratique de l'activité physique chez les étudiants du collégial. Canadian Journal of Behavioural Science / Revue canadienne des sciences du comportement. https://doi.org/10.1037/a0030221

Lemoyne, J., Valois, P. \& Guay, F. (2015). Physical Self-concept and Participation in Physical Activity in College Students. Medicine and Science in Sport and Exercise, 47(1), 142-150. doi: 10.1249/MSS.0000000000000378.

Lentillon-Kaestner, V., \& Roure, C. (2019). Coeducational and single-sex physical education : Students' situational interest in learning tasks centred on technical skills. Physical Education \& Sport Pedagogy, 24(3), 287-300. https://doi.org/10.1080/17408989.2019.1571186

Leriche, J. \& Walczak, F. (2014). Les obstacles à la pratique sportive des cégépiens. Cegep de Sherbrooke. https://cdc.qc.ca/parea/033137-leriche-walczak-obstaclespratique-sportive-cegepiens-sherbrooke-trois-rivieres-PAREA-2014.pdf

Leriche, J. \& Walczak, F. (2016). La perception des enseignants d'éducation physique au regard de leurs interventions. Article de vulgarisation. https://mobile.eduq.info/xmlui/bitstream/handle/11515/34602/leriche-walczakperception-enseignants-ed-phys-sherbrooke-trois-rivieres-article-PAREA2016. pdf? sequence $=2 \&$ isAllowed $=y$ 
eJRIEPS 50 janvier 2022

López-Pastor, V. M., Kirk, D., Lorente-Catalán, E., MacPhail, A. \& Macdonald, D. (2013). Alternative assessment in physical education: A review of international literature. Sport, Education and Society, 18(1), 57-76. https://doi.org/10.1080/13573322.2012.713860

Losier, G. F., Vallerand, R. J. \& Blais, M. R. (1993). Construction et validation de l'Échelle des Perceptions de Compétence dans les Domaines de Vie (EPCDV). Science et Comportement, 23(1), 1-16.

Martins, J., Marques, A., Sarmento, H. \& Costa, F. (2015). Adolescents' perspectives on the barriers and facilitators of physical activity: A systematic review of qualitative studies. Health Education Research, 30. https://doi.org/10.1093/her/cyv042

Menschik, D., Ahmed, S., Alexander, M. H. \& Blum, R. W. (2008). Adolescent physical activities as predictors of young adult weight. Archives of Pediatrics \& Adolescent Medicine, 162(1), 29-33. https://doi.org/10.1001/archpediatrics.2007.14

Mercier, K., \& Silverman, S. (2014). High School Students' Attitudes Toward Fitness Testing. Journal of Teaching in Physical Education, 33, 269-281. https://doi.org/10.1123/jtpe.2013-0153

Messier, C. (2016). Conception d'une épreuve terminale de cours pour évaluer une compétence en éducation physique au collégial [Essai, Université de Sherbrooke]. http://hdl.handle.net/11143/9634

Ministère de l'Éducation et de l'Enseignement supérieur du Québec (MEES). (2021). Le système scolaire québécois. http://www.education.gouv.qc.ca/references/etudierau-quebec/systeme-scolaire-quebecois/

Ministère de l'Éducation et de l'Enseignement supérieur (MEES). (2019). Plan stratégique 2019-2023.

https://cdn-contenu.quebec.ca/cdncontenu/adm/min/education/publications-adm/plan-strategique/plan-strategique2019-2023.PDF?1575660315

Ministère de l'Éducation et de l'Enseignement supérieur (MEES). (2016). Composantes de la formation générale - Extraits des programmes d'études conduisant au diplôme d'études collégiales (DEC). 52.

http://www.education.gouv.qc.ca/fileadmin/site web/documents/enseignementsuperieur/collegial/Composantes formation generale VF.pdf

Ministère de l'Enseignement supérieur, de la Recherche, de la Sciences et de la Technologie (MERST). (2013). Pour un virage santé à l'enseignement supérieur: Bilan 2007-2011 de la mise en œuvre du Cadre de référence pour une saine 


\section{eJRIEPS 50 janvier 2022}

alimentation et un mode de vie physiquement actif à l'enseignement supérieur http://www.education.gouv.qc.ca/fileadmin/site web/documents/enseignementsuperieur/Virage sante ES Bilan 2007-2011.pdf

Mueller, N., Rojas-Rueda, D., Cole-Hunter, T., de Nazelle, A., Dons, E., Gerike, R., Götschi, T., Int Panis, L., Kahlmeier, S., \& Nieuwenhuijsen, M. (2015). Health impact assessment of active transportation: A systematic review. Preventive Medicine, 76, 103-114. https://doi.org/10.1016/j.ypmed.2015.04.010

Nolin, B. et Institut national de santé publique du Québec. (2018). Indice d'activité physique. Enquête québécoise sur la santé des jeunes du secondaire 2016-2017. http://collections.banq.qc.ca/ark:/52327/3556385

Organisation mondiale de la santé. (2019a). D'après une nouvelle étude dirigée par l'OMS, la majorité des adolescents du monde ne sont pas assez actifs physiquement, ce qui met en danger leur santé actuelle et future. https://www.who.int/fr/news/item/2211-2019-new-who-led-study-says-majority-of-adolescents-worldwide-are-notsufficiently-physically-active-putting-their-current-and-future-health-at-risk Organisation mondiale de la santé. (2019b). Plan d'action mondial de l'OMS pour promouvoir l'activité physique 2018-2030: des personnes plus actives pour un monde plus sain.

https://apps.who.int/iris/bitstream/handle/10665/327168/9789242514186fre.pdf? sequence $=1$ \&is Allowed $=y$

Pasco, D. \& Spreux, D. (2014). La Motivation en Situation. Une Revue de Questions en Education Physique. eJRIEPS. Ejournal de la recherche sur l'intervention en éducation physique et sport, 31. https://doi.org/10.4000/ejrieps.2304

Patall, E. A. (2013). Constructing Motivation Through Choice, Interest, and Interestingness. Journal of Educational Psychology, 105(2), 522-534. https://doi.org/10.1037/a0030307

Patall, E. A. \& Yang Hooper, S. (2019). The Promise and Peril of Choosing for Motivation and Learning. Dans K. Ann Renninger \& S. E. Hidi (Éds.), The Cambridge Handbook of Motivation and Learning (p. 238- 262). Cambridge University Press.

Pollock, M. L., Gaesser, G. A., Butcher, J. D., Després, J-P., Dishman, R. K., Franklin, B. A. \& Garber, C. E. (1998). ACSM Position Stand. Medicine \& Science in Sports \& Exercise, $\quad 30(6), \quad$ 975-991. https://journals.Iww.com/acsmmsse/Fulltext/1998/06000/ACSM Position Stand The Recommended Quantity and.32.aspx 


\section{eJRIEPS 50 janvier 2022}

Reeve, J., Nix, G. \& Hamm, D. (2003). Testing Models of the Experience of SelfDetermination in Intrinsic Motivation and the Conundrum of Choice. Journal of Educational Psychology, 95(2), 375-392. https://doi.org/10.1037/0022$\underline{0663.95 .2 .375}$

Renninger, K. A. \& Hidi, S. (2019). Interest Development and Learning. In A. K. Renninger \& S. E. Hidi (Éds.), The Cambridge Handbook of Motivation and Learning (p. 265-290). Cambridge University Press.

Renninger, A. K. \& Hidi, S. (2016). The Power of Interest for Motivation and engagement. Routledge.

Robitaille, M.-P. (2019). Perceptions d'élèves du secondaire au sujet de leurs cours d'éducation physique. Mémoire de maîtrise. Université Laval. Québec. https://corpus.ulaval.ca/ispui/bitstream/20.500.11794/33911/1/34833.pdf

Roure, C., Kermarrec, G., \& Pasco, D. (2019). Effects of situational interest dimensions on students' learning strategies in physical education. European Physical Education Review, 25(2), 327-340.

Roure, C., Lentillon-Kaestner, V., Méard, J., \& Pasco, D. (2019). Universality and Uniqueness of Students' Situational Interest in Physical Education : A Comparative Study. Psychologica Belgica, 59(1), 1-15. https://doi.org/10.5334/pb.446

Roure, C., Méard, J., Lentillon-Kaestner, V., Flamme, X., Devillers, Y., \& Dupont, J.-P. (2019). The effects of video feedback on students' situational interest in gymnastics. Technology, Pedagogy and Education, 28(5), 563-574. https://doi.org/10.1080/1475939X.2019.1682652

Roure, C., \& Pasco, D. (2020). Le pouvoir de l'intérêt en situation en éducation physique : un modèle pour concevoir des situations d'apprentissage motivantes. Dans V. Lentillon-Kaesther (Ed.), Penser l'éducation physique autrement (p. 15-32). Louvain-la-Neuve, Belgique: EME Editions. http://hdl.handle.net/20.500.12162/4272

Roure, C., \& Pasco, D. (2018). Exploring situational interest sources in the French physical education context. European Physical Education Review, 24(1), 3- 20.

Roure, C. \& Pasco, D. (2017). Impact de la conception d'une situation d'apprentissage en badminton sur l'intérêt en situation d'élèves du secondaire. eJRIEPS. Ejournal de la recherche sur l'intervention en éducation physique et sport, 42. https://doi.org/10.4000/ejrieps.533 


\section{eJRIEPS 50 janvier 2022}

Ryan, R. M. \& Deci, E. L. (2000). Intrinsic and Extrinsic Motivations: Classic Definitions and New Directions. Contemporary Educational Psychology, 25(1), 54-67. https://doi.org/10.1006/ceps.1999.1020

Standage, M., Gillison, F. B., Ntoumanis, N., \& Treasure, D. C. (2012). Predicting Students' Physical Activity and Health-Related Well-Being: A Prospective Cross-Domain Investigation of Motivation Across School Physical Education and Exercise Settings. Journal of Sport and Exercise Psychology, 34(1), 37-60. https://doi.org/10.1123/jsep.34.1.37

Taylor, I. M., Ntoumanis, N., Standage, M., \& Spray, C. M. (2010). Motivational predictors of physical education students' effort, exercise intentions, and leisure-time physical activity: A multilevel linear growth analysis. Journal of Sport and Exercise Psychology, 32(1), 99-120.

Trost, S. G. \& Loprinzi, P. D. (2008). Exercise-Promoting healthy lifestyles in children and adolescents. Journal of Clinical Lipidology, 2(3), 162-168. https://doi.org/10.1016/j.jacl.2008.03.001

Université de Sherbrooke. (2018). Chaire de recherche Kino-Québec sur l'adoption d'un mode de vie physiquement actif en contexte scolaire. https://www.usherbrooke.ca/recherche/repertoire-de-projets-derecherche/projet/511/

Viau, R. (2009). La motivation à apprendre en milieu scolaire. Éditions du renouveau pédagogique (ERPI).

Viau, R. \& Bouchard, J. (2000). Validation d'un modèle de dynamique motivationnelle auprès d'élèves du secondaire. Canadian Journal of Education/Revue canadienne de l'éducation, 25(1), 16-26-16-26.

Viau, R., Joly, J. \& Bédard, D. (2004). La motivation des étudiants en formation des maîtres à l'égard d'activités pédagogiques innovatrices. Revue des sciences de l'éducation, 30(1), 163-176. https://doi.org/10.7202/011775ar

Wiersma, L. D. \& Sherman, C. P. (2008). The Responsible Use of Youth Fitness Testing to Enhance Student Motivation, Enjoyment, and Performance. Measurement in Physical Education and Exercise Science, 12(3), 167-183. https://doi.org/10.1080/10913670802216148

Wigfield, A. \& Eccles, J. S. (2000). Expectancy-Value Theory of Achievement Motivation. Contemporary Educational Psychology, 25(1), 68-81. https://doi.org/10.1006/ceps.1999.1015 
eJRIEPS 50 janvier 2022

Note des autrices:

Cette étude a été menée dans le cadre d'une recherche financée par le Programme d'aide à la recherche sur l'enseignement et l'apprentissage (PAREA) du ministère de l'Éducation et de l'Enseignement supérieur (MEES) du Québec (\# 10670). 